\title{
A step-by-step analysis of the polishing process for tungsten specimens
}

\author{
Armin Manhard*, Gabriele Matern and Martin Balden
}

Max-Planck-Institut für Plasmaphysik, EURATOM Association, Boltzmannstraße 2, 85748

Garching, Germany

*corresponding author: armin.manhard@ipp.mpg.de

\section{Keywords:}

tungsten, polishing, cross-section, focused ion beam (FIB), scanning electron microscopy (SEM), deformation layer

\begin{abstract}
The different stages of the polishing process of polycrystalline tungsten samples were investigated by scanning electron microscopy of both the sample surface and of cross-sections prepared with the help of a focused ion beam. It is shown that a distortion layer is present at the sample surface after mechanical fine grinding and even after polishing with diamond suspension, although the sample has a mirror-like finish. A sufficiently long chemomechanical polishing step using an alkaline colloidal silica suspension was able to remove this distortion layer. Although electropolishing produced an even smoother surface, the microstructure quality after chemo-mechanical polishing is comparable to that of an electropolished sample.
\end{abstract}

\section{Introduction}

Tungsten is a promising material for the plasma-facing wall in future nuclear fusion reactors. In the environment of such a fusion reactor, the wall is subjected to large fluxes of deuterium and tritium ions with typical energies between several eV and several $100 \mathrm{eV}$. For safety reasons, it is important to estimate the amount of radioactive tritium that can be stored in plasma-facing components due to ion implantation [1]. Therefore, tungsten specimens are exposed to deuterium plasmas or deuterium ion beams under laboratory conditions to study the interaction of hydrogen isotopes with tungsten surfaces. In order to perform such experiments under controlled conditions, it is important to use specimens with well-defined surfaces, in particular since the stopping range of hydrogen isotope ions at energies relevant for fusion reactor applications (see above) is short, between a few $\mathrm{nm}$ and several $10 \mathrm{~nm}$.

It is generally known to metallographers that mechanical polishing of metals can lead to deformation layers at the surface (see, e.g. [2]), in particular for soft metals such as copper. Nevertheless, this is also observed for tungsten [3]. Apart from obscuring the true bulk 
microstructure during, e.g., scanning electron microscopy (SEM) analysis, such a deformation layer could have a large influence on the deuterium retention, which can depend strongly on the microstructure of the tungsten samples [4]. There is also experimental evidence that a deformed surface layer can influence, e.g., the formation of blisters on deuterium-plasmaexposed tungsten [3]. There are numerous methods for removing this deformation layer, e.g., electropolishing [2]. For the work presented here, a final chemo-mechanical polishing step using an alkaline colloidal silica suspension was applied.

The aim of the work presented here was to optimise the polishing procedure for tungsten specimens. Therefore, a set of specimens was first ground with increasingly fine SiC abrasive paper (P400 up to $\mathrm{P} 4000$ ). The polishing was then continued with $1 \mu \mathrm{m}$ diamond suspension, followed by the final chemo-mechanical step. At several intermediate steps of the process individual specimens were removed in order to study the effect of the polishing procedure up to that point. In the following, the specimens were analysed with a FEI HELIOS NanoLab 600 dual-beam scanning microscope, which is equipped both with an electron beam for imaging and a focussed ion beam (FIB) for in situ cross-section preparation. To complete the picture, roughness measurements were performed with an Olympus LEXT OLS4000 confocal laser scanning microscope.

\section{Experimental}

The specimens used in the experiments presented here were hot-rolled tungsten with a nominal purity of $99.97 \mathrm{wt} . \%$ and manufactured by PLANSEE. They had a thickness of $0.8 \mathrm{~mm}$ and were cut into rectangular pieces of $12 \mathrm{x} 15 \mathrm{~mm}^{2}$. All specimens were from one single manufacturing batch and can therefore be considered to be identical.

The first specimen was removed after the final grinding step with P4000 SiC paper. This step was performed with a force of $20 \mathrm{~N}$ for 1 minute. Subsequently, polishing with diamond suspension was performed with a force of $35 \mathrm{~N}$ on a textile pad for 10 minutes. After this treatment, the second specimen was removed. The remaining specimens were chemomechanically polished with alkaline colloidal silica suspension (Logitech "SF1") for 10, 20 and 30 minutes, respectively. For this polishing step a synthetic felt pad (Logitech "SUBA$\mathrm{X}^{\prime \prime}$ ) was used, the applied force was $30 \mathrm{~N}$. The specimens were then all cleaned with isopropanol in an ultrasonic bath in order to remove any residual polishing agent or debris. For comparison, an additional specimen was electropolished for 2-3 minutes with 2.5\% $\mathrm{NaOH}$ solution in water after grinding. The polishing voltage was $25 \mathrm{~V}$, the electrolyte temperature was stabilised at $20^{\circ} \mathrm{C}$. The specimen was then heated to $1200 \mathrm{~K}$ in vacuum to remove any residual oxide layer that formed during electropolishing. The grain structure of the sample did not change during this heat treatment.

On each specimen, plan view images of the surface as well as cross-section images of a FIB cut parallel to the rolling direction were acquired. The images were typically recorded with a secondary electron detector. It should be noted at this point that the orientation contrast visible in SEM images does not allow discerning between small-angle and large-angle grain boundaries. Taking into account the fabrication method of the specimen (i.e., hot rolling), it can be expected that most of the grains visible in the images are separated by small-angle grain boundaries and are, accordingly, actually sub-grains, as it was reported, e.g., for hotrolled molybdenum [5].

All surface images presented here were acquired with the specimen surface normal parallel to the electron beam. Prior to the FIB cut, a protective layer of an amorphous Pt-C compound was deposited in situ on the specimen surface by locally decomposing a precursor gas with the help of the electron beam. The electron beam was chosen for the deposition instead of the 
ion beam in order to avoid any artefacts produced by ions impinging on the unprotected surface during the initial phase of the layer deposition. This layer is visible as a dark, slightly structured area at the top of all cross-section images presented here. For cutting and imaging of the cross-section, the specimen surface normal was tilted by $52^{\circ}$ with respect to the electron beam. This corresponds to normal incidence of the ion beam. All cross-section images are presented without a geometric tilt correction for the $38^{\circ}$ inclination of the crosssectional plane towards the electron beam.

For each of the samples, a roughness analysis was performed on a $256 \times 256 \mu \mathrm{m}^{2}$ scan acquired by the confocal laser scanning microscope. This image size corresponds roughly to the lower end magnifications typically used for SEM analysis of the grain structure. For example, the surface SEM images presented here have a full width of $25.6 \mu \mathrm{m}$. RMS roughness values $R_{q}$ were calculated for 3 horizontal, 3 vertical and 2 diagonal profiles of the 3-D surface maps. The values given for each sample are averaged over the values for these 8 profiles. The error margins correspond to the standard deviations of each such set of roughness measurements.

\section{Results}

The specimen that was removed after grinding (see Figure 1) shows numerous scratches, pits and cracks, and an apparently very fine grain structure on the surface (a). The cross-section image (b) reveals that this is due to a top layer of about $0.5 \mu \mathrm{m}$ where the grains have fragmented. Deeper into the bulk, the grains are much larger. After diamond polishing, the specimen surface appears already mirror-like to the naked eye. During this step, the nearsurface layer of strongly fragmented grains is mostly removed (see Figure 2). However, a clear grain structure is not yet discernible in the surface SEM image (a). The grains at the surface accordingly still have to be considered as heavily deformed. The deformation becomes especially obvious when comparing the top layer of grains with bulk grains in the cross-section images (b). Also, both the surface and the cross-section view reveal that the specimen surface still contains many cracks, pores and small craters. It can therefore already be concluded that diamond polishing alone is insufficient for producing specimens where the near-surface grains have the same properties as the bulk grains.

The following chemo-mechanical polishing step then gradually removes the damage that remains after polishing with diamond suspension. However, at least 30 minutes of this treatment are necessary to fully remove all traces of a deformation layer. A clear grain structure is then visible on the specimen surface (see Figure 3a), with only a small number of scratch traces. The cross-section image (Figure $3 \mathrm{~b}$ ) shows that the top layer of grains is now indeed representative for the bulk of the specimen. The slight deformations visible in some surface grains occur in bulk grains as well.

Surface (Figure 4a) and cross-section (Figure 4b) images of the electropolished specimen look remarkably similar to those of the specimen that was chemo-mechanically polished for 30 minutes. Only some grain boundaries show slightly enhanced contrast in the surface image of the surface (Figure 4). This can be attributed to slight preferential etching of grain boundaries intersecting with the surface. As for the chemo-mechanically polished specimen, there is also no discernible difference between bulk grains and grains adjacent to the specimen surface. This provides further evidence that the remaining distortions, which are visible in some grains as different grayscale shadings, are not preparation artefacts. They are rather caused by the hot-rolling process and are therefore an intrinsic property of the specimens. Accordingly, the chemo-mechanical polishing process presented here is indeed able to produce specimens with a surface quality that is comparable to the result of electrochemical polishing. 
Roughness analyses of the samples after the different polishing treatments show that after removing the large initial surface roughness by grinding with SiC paper up to P4000, the RMS roughness $R_{q}$ is reduced from $R_{q}=22 \pm 5 \mathrm{~nm}$ to $R_{q}=17 \pm 3 \mathrm{~nm}$ by polishing with diamond suspension. 10 minutes of chemo-mechanical polishing reduces the roughness even further to $R_{q}=10 \pm 2 \mathrm{~nm}$. For further chemo-mechanical polishing, the roughness barely changes anymore and stagnates at about $R_{q}=9 \pm 2 \mathrm{~nm}$ for 20 minutes respectively $R_{q}=9 \pm 1$ $\mathrm{nm}$ for 30 minutes. During this final chemo-mechanical polishing treatment, only the internal microstructure of the surface is refined by removing the distortion layer caused by the previous polishing steps, as it was already discussed above. Only by electropolishing, even smoother surfaces could be produced. Here an RMS value of $\mathrm{R}_{\mathrm{q}}=3 \pm 1 \mathrm{~nm}$ was achieved. This value may still be overestimated since it is close to the resolution limit of confocal microscopy and some measurement artefacts were already visible on the image.

Compared to electropolishing, the chemo-mechanical polishing method presented here has the advantage of being highly reproducible and can be applied to multiple samples at the same time with a suitable polishing machine. With the equipment used for this study, up to 6 samples could be polished at the same time, which allows for a quick production of a large number of identical samples. Electropolishing, on the other hand, was found to produce reliable results only if the samples were embedded in epoxy resin in order to prevent any contact between the electrolyte and the anode current supply. The embedding of the samples in resin and particularly the removal of the resin after the electropolishing are tedious processes. They strongly increase the total time required to prepare one sample, thus making electropolishing unsuitable producing a large number (i.e., hundreds) of identical samples for systematic parameter studies. Yet, electropolishing may still be of merit if extremely smooth surfaces are required.

\section{Summary}

It was investigated by scanning electron microscopy on surfaces and cross-sections how mechanical and chemo-mechanical polishing affects the grain structure of tungsten at the polished surface, even if the visual appearance of the specimen is already mirror-like. It was demonstrated that grinding and diamond polishing produce both microstructures at the surface that are not equivalent to the bulk structure. These preparation artefacts can be removed by 30 minutes of chemo-mechanical polishing using an alkaline colloidal silica suspension. Although electropolishing can produce even smoother surfaces, the final near-surface microstructure then closely resembles that of an electropolished surface, where the grains adjacent to the surface are virtually indistinguishable from bulk grains. 


\section{Literature}

[1] Roth, J. et al.: J. Nucl. Mater. 390-391 (2009), 1-9

[2] Petzow, G.: Metallographisches Ätzen, 5. Auflage, Gebrüder Borntraeger, BerlinStuttgart, Germany, 1976, S. 8ff

[3] Alimov, V. Kh. et al.: J. Nucl. Mater 420 (2012) 1-3, 519-524

[4] Manhard, A. et al.: J. Nucl. Mater. 415 (2011) 1S, S632-S635

[5] Primig, S. et al.: Prakt. Metallogr. 48 (2011) 7, 345-355

\section{About the Authors}

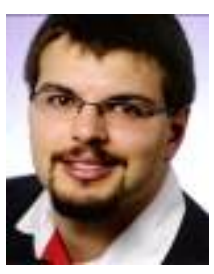

Armin Manhard has been working on his Ph.D. thesis from 2008-2011 and is now working as a postdoc at the Max-Planck-Institute for Plasma Physics in Garching. The focus of his research is the retention of deuterium in tungsten and its correlation with the microstructure and defect density of the tungsten.

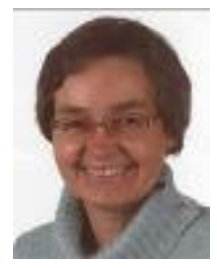

Gabriele Matern works as a metallographer at the Max-Planck-Institute for Plasma Physics in Garching. She performs sample preparation as well as optical and scanning electron microscopy analysis of material samples and various components, e.g., from the fusion experiment ASDEX Upgrade. 


\section{Figure captions}

\section{Figure 1}

Tungsten sample after grinding with SiC paper up to P4000. The surface image (a) shows many pits and scratches, and apparently very small grains. The cross-section image (b) reveals that the grains in a top layer of about $0.5 \mu \mathrm{m}$ thickness have fragmented into many small grains, while the bulk grains are much larger.

\section{Figure 2}

Tungsten sample after 10 minutes of polishing with a diamond suspension. The surface image shows no clear grain structure, but still many pits and scratches. The cross-section view (b) clearly shows that the top layer of grains is heavily deformed compared to bulk grains. Cracks and pores are also visible.

\section{Figure 3}

Tungsten sample after 30 minutes of chemo-mechanical polishing with an alkaline colloidal silica suspension. Grains are now clearly visible in the surface image (a), and only a few scratch traces remain. The grains adjacent to the surface and deeper in the bulk are virtually indistinguishable in the cross-section view (b).

\section{Figure 4}

Tungsten sample electropolished for 2-3 minutes in $1.5 \mathrm{wt} . \% \mathrm{NaOH}$ solution. The sample was subsequently annealed for 60 minutes at $1200 \mathrm{~K}$ in a high vacuum oven to remove any residual oxide layer. Both the surface (a) and cross-section (b) images are equivalent to those from the chemo-mechanically polished sample in Figure 3.

\section{Figure 5}

RMS roughness values $R_{q}$ of tungsten samples after different polishing treatments. The roughness is continuously reduced from the grinding step over diamond polishing up to 10 minutes of chemo-mechanical polishing. After that, the roughness is barely affected anymore. Only electropolishing (here: after fine grinding) can produce even smoother surfaces. 


\section{Figures}

Figure 1
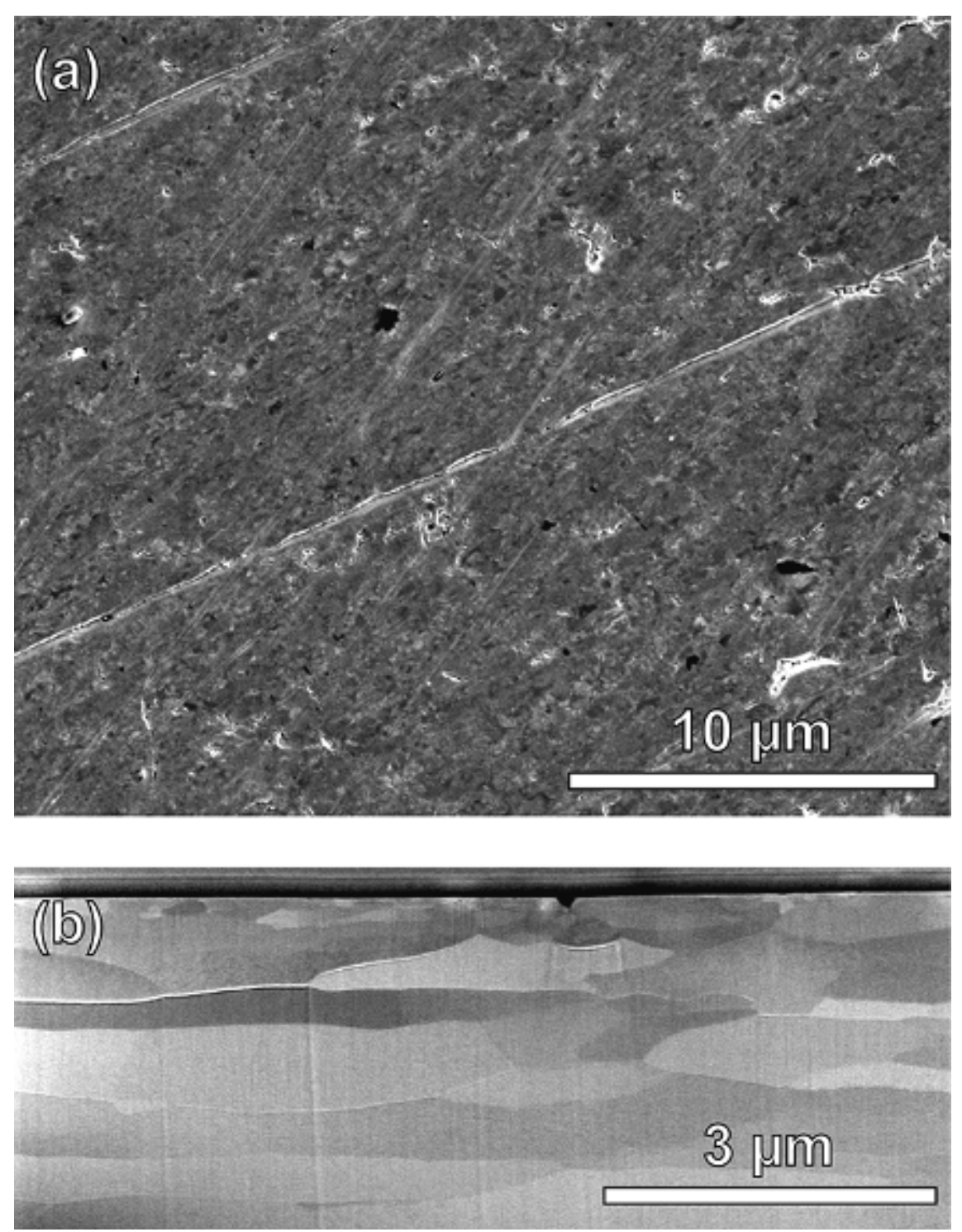
Figure 2
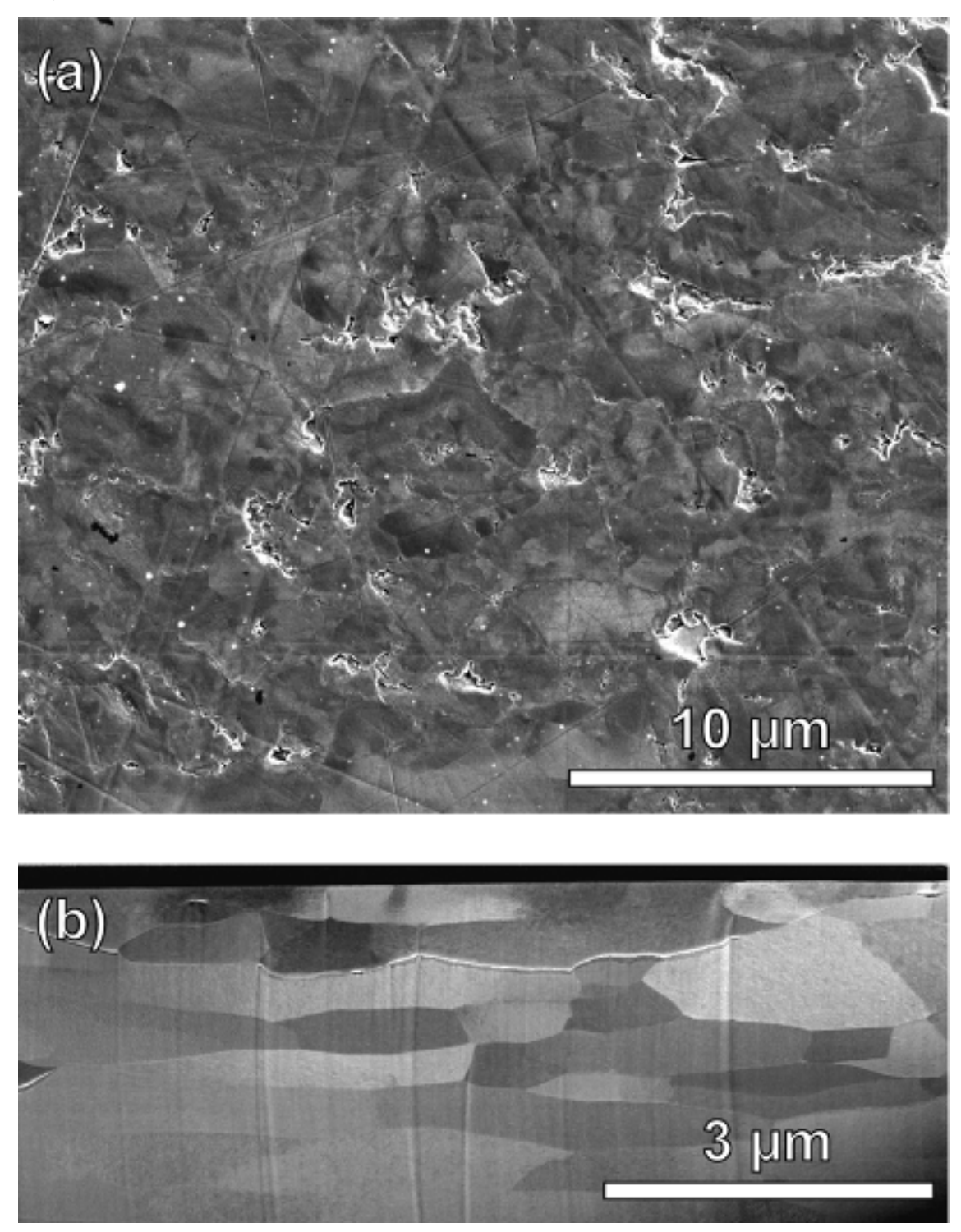
Figure 3
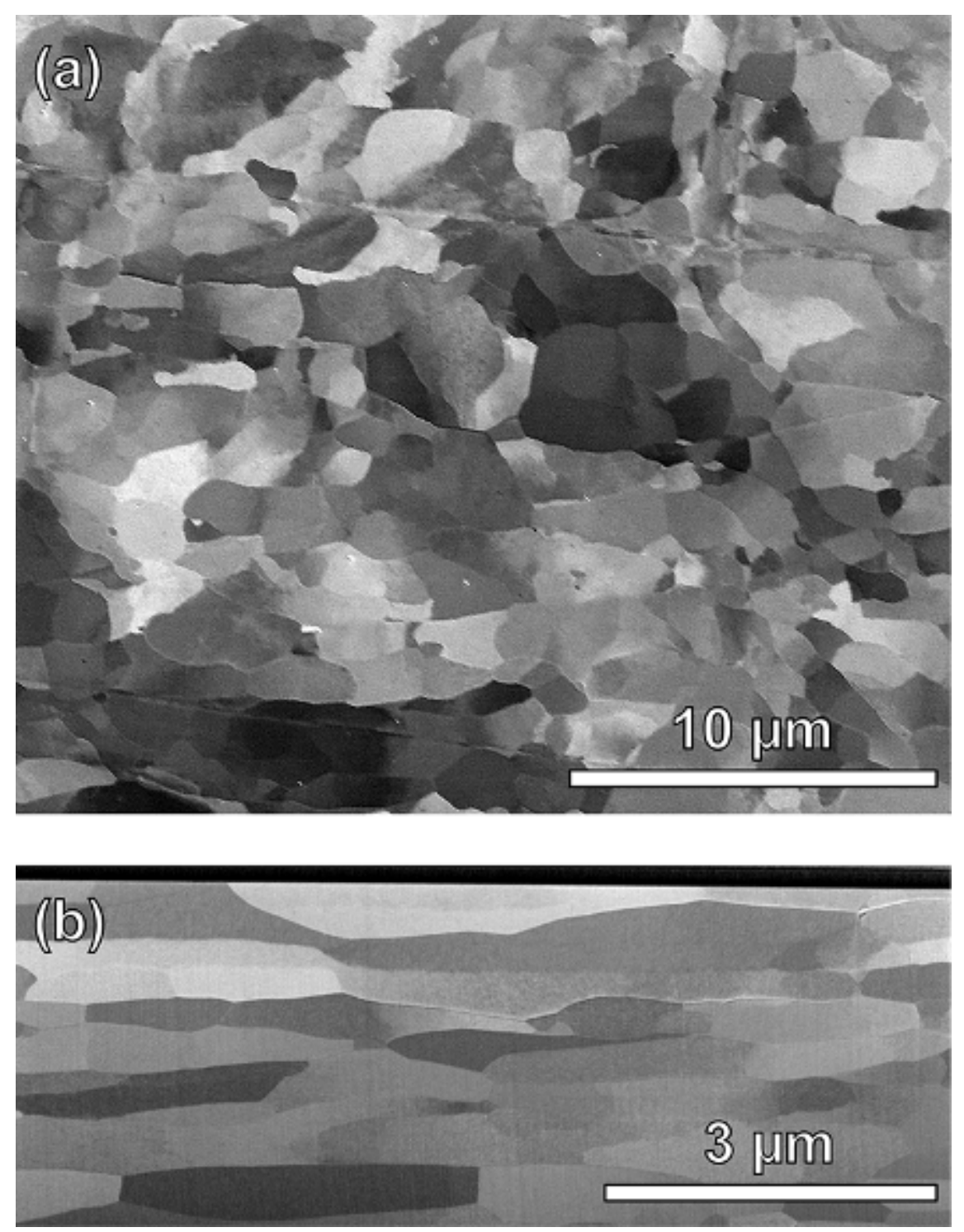
Figure 4
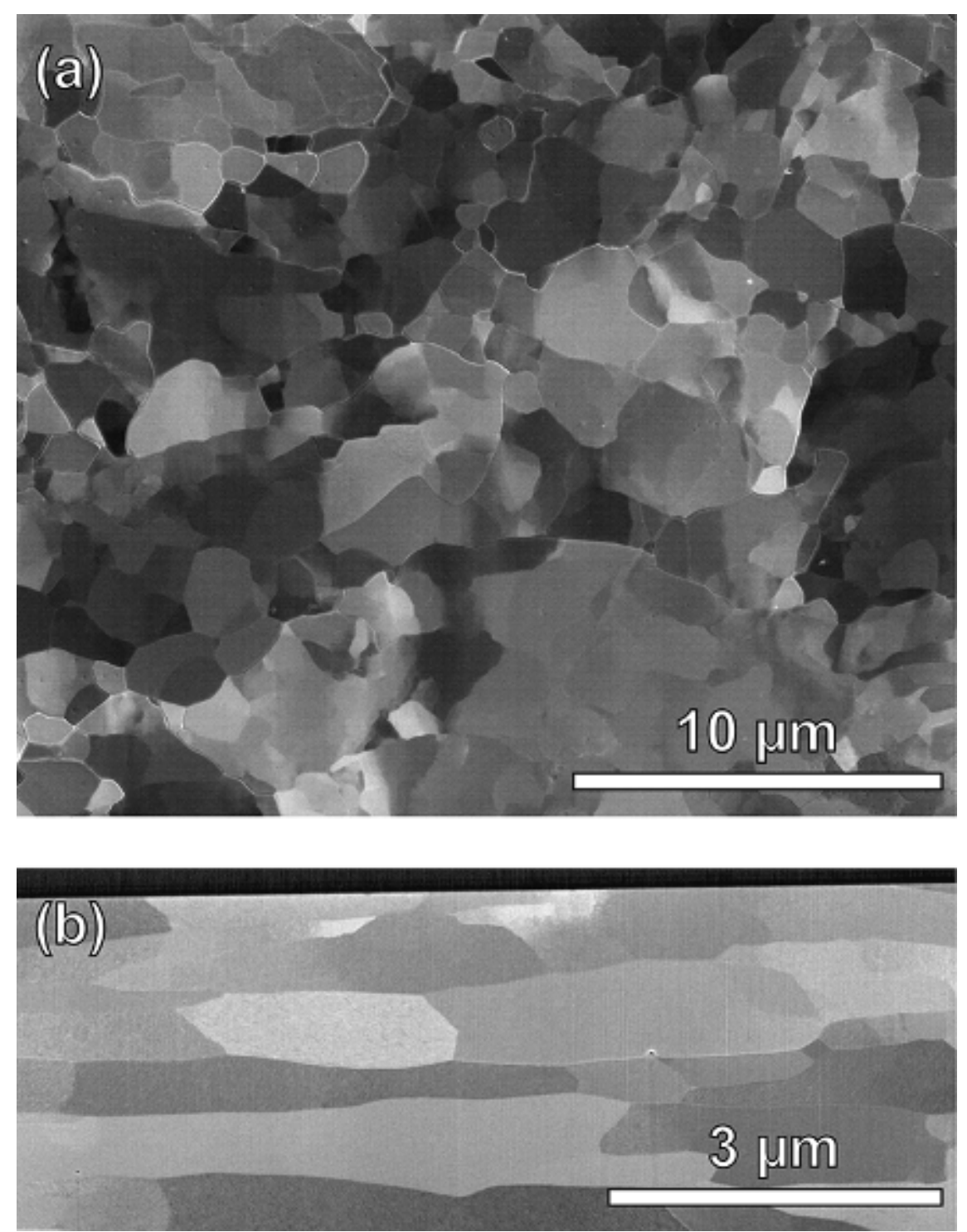
Figure 5

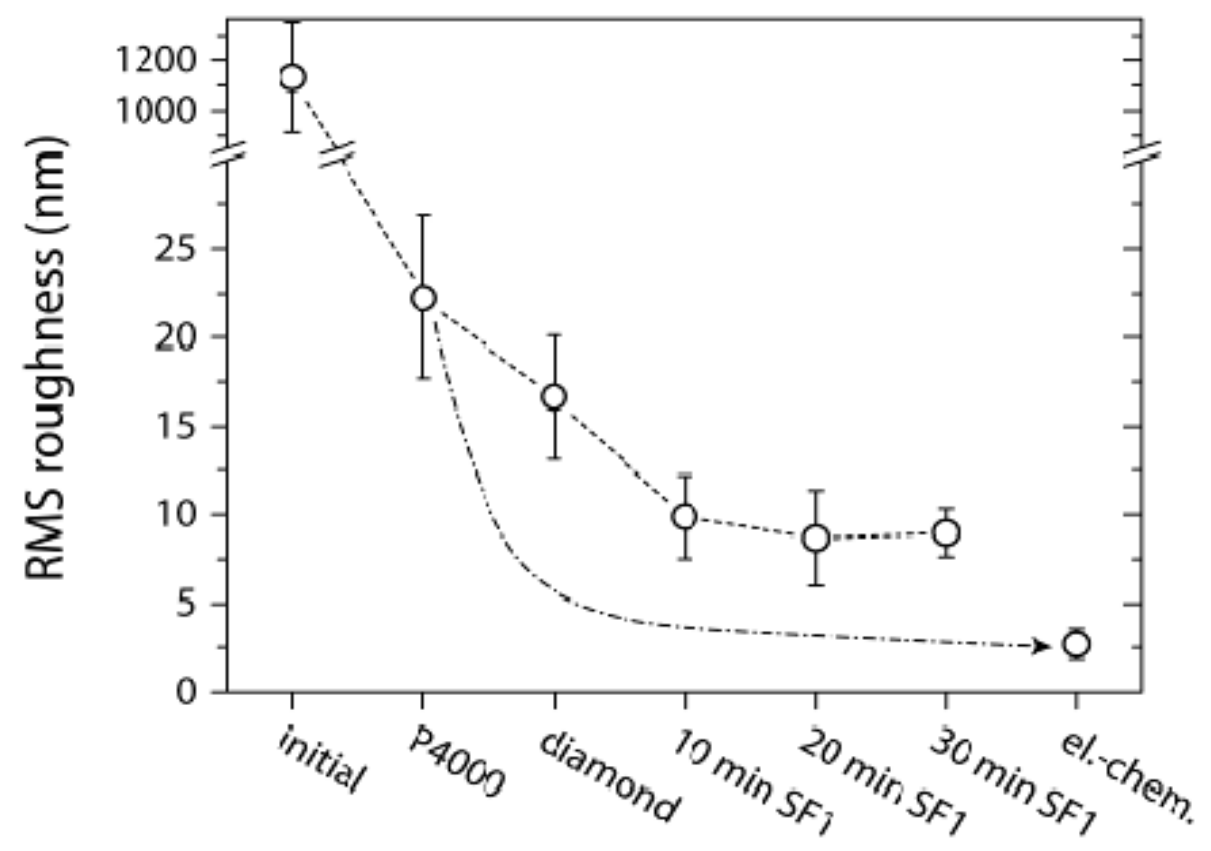

